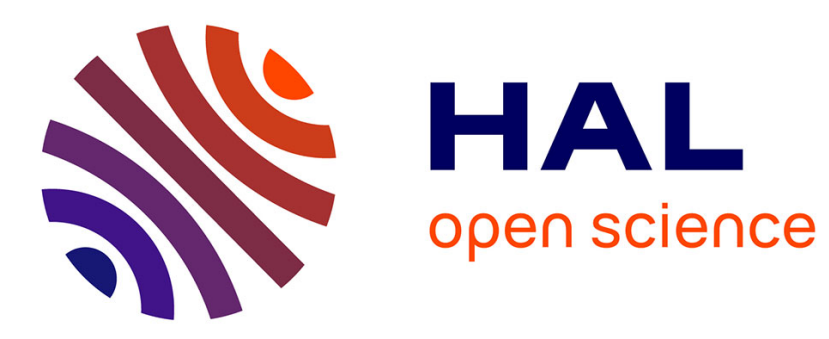

\title{
Key Characteristics identification by global sensitivity analysis
}

Dana Idriss, Pierre Beaurepaire, Lazhar Homri, Nicolas Gayton

\section{To cite this version:}

Dana Idriss, Pierre Beaurepaire, Lazhar Homri, Nicolas Gayton. Key Characteristics identification by global sensitivity analysis. International Journal on Interactive Design and Manufacturing, 2019, pp.1-12. 10.1007/s12008-019-00625-z . hal-02379193

\section{HAL Id: hal-02379193 \\ https://hal.science/hal-02379193}

Submitted on 25 Nov 2019

HAL is a multi-disciplinary open access archive for the deposit and dissemination of scientific research documents, whether they are published or not. The documents may come from teaching and research institutions in France or abroad, or from public or private research centers.
L'archive ouverte pluridisciplinaire HAL, est destinée au dépôt et à la diffusion de documents scientifiques de niveau recherche, publiés ou non, émanant des établissements d'enseignement et de recherche français ou étrangers, des laboratoires publics ou privés. 


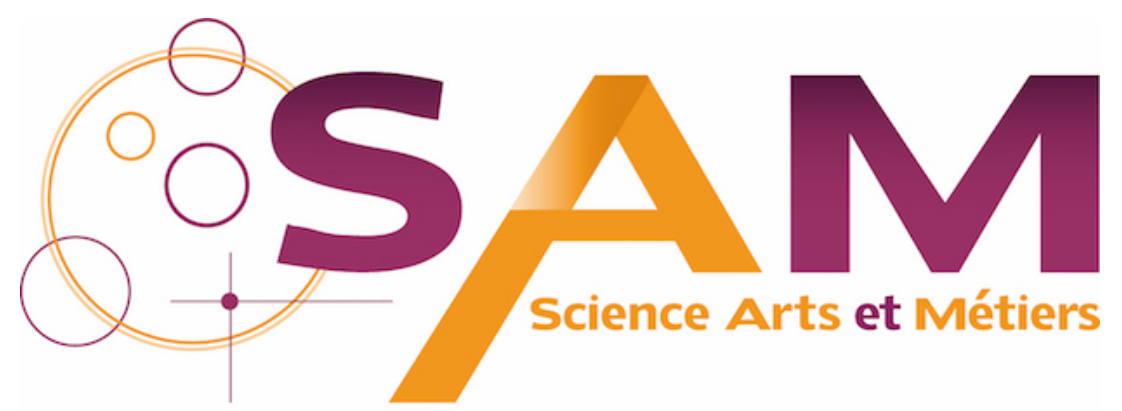

Archive Ouverte - Open Repository

\section{Science Arts \& Métiers (SAM)}

is an open access repository that collects the work of Arts et Métiers ParisTech researchers and makes it freely available over the web where possible.

This is an author-deposited version published in: https://sam.ensam.eu Handle ID: .http://hdl.handle.net/null

\section{To cite this version :}

Dana IDRISS, Pierre BEAUREPAIRE, Lazhar HOMRI, Nicolas GAYTON - Key Characteristics identification by global sensitivity analysis - International Journal on Interactive Design and Manufacturing (IJIDeM) p.1-12 - 2019 


\section{Key Characteristics Identification by Global Sensitivity Analysis}

Received: date / Accepted: date

\section{Introduction}

Manufacturing of products is a process subjected to uncertainties which are associated with the geometrical imperfections that the products are subjected to. The imperfections cause unfavourable effects on the performance of the system. To limit these effects it is preferred to take into account the uncertainties as soon as possible during the design stage. One of the main sources of uncertainty in products assembly, consisting of mating or interconnecting sub components, is the variation arising from manufacturing processes. Tolerancing is therefore an important part of design phase and a key element that should be considered when dealing with robustness of any product. Tolerancing decisions should respect the limited capabilities of the required manufacturing processes as well as the functionality of the product.

Designers and manufacturing engineers adopt new practices, in the mechanical and industrial engineering fields, in the early phases of the design to reach a product with high quality and minimum cost. This is known as interactive design [1]. The interactive design is also considered as a new practice in the early phases of the design to support decision making. In the interactive design, manufacturing a product can be controlled by three factors: the experts' knowledge, the end-user satisfaction and the realization of functions [2]. Ibrahim and Chassapis [3] present an interactive environment between the design model and the process capabilities to evaluate the risk of manufacturing variations. In the early design phase, the designers set the nominal dimensions, the tolerance intervals and the capability requirements that need to be respected in the manufacturing process. They also indicate the dimensions that have to be considered as KeyCharacteristics (KCs) on the engineering drawings, i.e. the

Address(es) of author(s) should be given dimensions with a first order influence on the functionality (sometimes indicated with a diamond, especially in the French automotive industry). This paper proposes a method that can be considered as part of interactive design methods. It aims to reduce the number of $\mathrm{KCs}$ in the engineering drawings during the design stage. Therefore, the cost of the production is reduced during the manufacturing process. As a consequence, the costumer can buy the product with a good quality and an affordable price.

Several definitions of the Key Characteristics can be found in the literature and standards such as the European standards EN 9100 and 9103. The Key Characteristics are defined in [4] as follows: "A Key Characteristic is a quantifiable feature of a product or its assemblies, parts, or processes whose expected variation from target has an unacceptable impact on the cost, performance, or safety of the product". Key Characteristics need special attention and efforts during their production. The larger the number of dimensions set as $\mathrm{KC}$ the higher is the cost of manufacturing. There is a need to reduce the number of KCs by identifying only the necessary ones. Different methods exist for the KCs' identification. They can be distinguished between qualitative and quantitative methods. The qualitative methods are based on the concept of the KC flow-down which is a method that can identify the low level Key Characteristics such as the sub-assemblies of the product [5]. However, the classification of the identified KCs is not possible when $\mathrm{KC}$ flow-down approach is used. In practice, the industries use the Failure Mode Effects and Criticality Analysis (FMECA), which is a common qualitative method used for the identification of critical chains, as a way to specify the KCs [6]. They assume that once a chain is set as critical, all the involved parts are set as KCs [7]. This is a very conservative way to identify the KCs since they may not all have the same impact. Some dimensions have first order impact and some others have reduced impacts on the 
performance of the system. The use of such methods cause higher costs of production. The lack of knowledge in the qualitative approaches pushed researches to adopt quantitative methods for the identification of KCs. Thornton [8] used the Taguchi loss function as a measure of the relative quality of the product-KC and proposed a variation model to calculate the impact of a $\mathrm{KC}$, which is dependent on the sensitivity to the variation of product quality. Dantan et al. [9] proposed an information model, based on KC-Flowdown and some quantitative information, to formalize and capitalize the causality between Manufacturing Process KCs and Part / Product KCs. According to Han et al. [10] who did a detailed review on the $\mathrm{KCs}$, both the qualitative and the quantitative methods are applicable to identify the key process characteristics.

The objective of this paper is to develop quantitative methods for the identification of the most influencing dimensions and classify them as KCs in the same critical functional requirement in the design stage. The approaches already mentioned for the identification of $\mathrm{KCs}$ do not provide the uncertain impacts of each dimension in the early design stage. Further analysis is needed for this purpose. This paper suggests the use of the sensitivity analysis in this context.

The sensitivity methods are used to study the influence of input parameters on the output of a model. They are distinguished between the local ones and the global ones. Different works exist in the literature where the sensitivity analysis is used in the context of tolerancing. Stuppy and Meerkamm [11] applied the arithmetical contributor analysis, statistical contributor analysis and high-low-median sensitivity analysis in tolerance management, which are local sensitivity analysis methods. Ledoux and Teissandier [12] proposed a method based on local sensitivities to analyse the influence of the tolerance zones and geometric parameters on the probability of non-compliant functional requirement. Eifler et al. [13] provided an approach to measure the impact of different design parameters in a network of physical effects, based on existing methods for sensitivity analysis. The different indices are studied with respect to their applicability during conceptual design. Ziegler and Wartzack [14] implemented global sensitivity analysis methods on convex hull based tolerancing techniques, such as deviation domains.

In this study, a methodology is proposed for the classification of the dimensions according to their influence on the performance of the system. It is inspired from the works previously discussed. The proposed approach is based on the sensitivity analysis and more precisely on the global sensitivity analysis. It allows the classification of the dimensions with high or reduced impact on the performance of the system, that is dimensions that are defined as $\mathrm{KC}$ or not KC. It quantifies the impact of the variation of the statistical parameters on the variation of the Non Conformity Rate (NCR) which is the probability that the functional re- quirement is not satisfied. The main contribution of the proposed approach is that it takes into account the variability of the statistical parameters corresponding to the dimensions, that is the variability of the corresponding distributions' parameters. This aspect is not taken into account in the previous works that perform the sensitivity analysis in the context of tolerancing. In addition, this method is applicable when the system comprises more than one functional requirement.This method also allows an interactive environment between the designers and manufacturing engineers by taking into account the uncertainties in tolerances in the design drawings and the manufacturing variations during the processes.

The outline of this paper is as follows: Section 2 describes the uncertainties in parameters and how they propagate to the NCR; Section 3 describes the followed procedure in this article and explains the choices of the model components. Then the different steps of the approach with the mathematical formulations are given in Sect.4; Section 5 presents the case studies in which the proposed method is applied. Finally, conclusions and perspectives are developed in Sect. 6.

\section{Propagation of uncertainties}

\subsection{Functional requirements and Non Conformity Rate}

In industry, the quality of the product is one of the main measures checked to satisfy the needs of the customer. According to the technical requirements and specifications imposed by the customer, a list of functional requirements is defined for dimensional chains. A functional requirement can be expressed as a function of input parameters $\mathbf{X}$, these parameters are the dimensions of the parts included in the dimensional chains. In the case of a function $f$, the dimensional chain is expressed as:

$Y=f(\mathbf{X})$

where $\mathbf{X}$ is the vector defining the dimensions. The mathematical formulation of this function can be linear such as the case of a linear stack-up or non-linear for problems of greater complexity. Furthermore, $f$ can be implicit when the system studied is over-constrained [15]. Tolerance analysis is used to improve the product quality and to reduce the manufacturing cost in the design stage [16]. The value of functional requirements is verified by the tolerance analysis after setting tolerances on each dimension. When the functional characteristic of a product does not respect the functional specified requirements, it is called a defect or a non conform product. The defect probability is defined as the probability that the functional characteristic does not respect the specification limits. In this paper, the defect probability is 
referred to as the Non Conformity Rate (NCR). The NCR therefore provides suitable metrics to measure the quality. It can be interpreted as the probability that an assembly is non-functional, or as the frequency of manufacturing nonfunctional components. The NCR, defined by Eq.(2), is the probability that a functional requirement is outside the permissible bounds. It is often expressed in parts per million (ppm).

$N C R=\mathrm{P}\left(Y \notin\left[L S L_{Y} ; U S L_{Y}\right]\right)$

$L S L_{Y}$ and $U S L_{Y}$ are respectively the lower specification limit and the upper specification limit. They are called the functional bounds.

\subsection{Dimensions' uncertainty}

The manufacturing process vary from day to another depending on the controlling factors, so the characteristics will deviate from the required specifications. The process capability ratio $C_{p}$ is a quantitative way to express the process capability [17]. Another process capability ratio for off-centre process is $C_{p k}$, it takes into account the location of the process mean with respect to the specifications. The reference standard ISO 22514-1 provides definitions and mathematical expressions for the capability indices $C_{p}$ and $C_{p k}$ that are applicable for all types of distributions. When the process is in a state of statistical control, $C_{p}$ is expressed as the ratio of the tolerance interval $t$ and the reference interval. The normal distribution is frequently assumed for the dimensions or the characteristics of a statistical sample, being simple to implement and close to the real cases of the production [4]. For the case of a normal distribution the capability requirements are expressed such as Eqs. (3-4).

$C_{p}=\frac{t}{6 \sigma}$

$C_{p k}=\frac{t / 2-|\delta|}{3 \sigma}$

where $\delta$ is the mean shift of the off-centred distributions and $\sigma$ is the corresponding standard deviation. The process in this paper is assumed to be in statistical control, so the capability indices $C_{p}$ and $C_{p k}$ are used. The capability requirements to be respected are $C_{p} \geq C_{p}^{(r)}$ and $C_{p k} \geq C_{p k}^{(r)}$. $C_{p}^{(r)}$ and $C_{p k}^{(r)}$ are industrial requirements usually imposed by the companies. A capability domain $V_{D}$ is defined for each part dimension $X$ which defines the set of acceptable $(\delta, \sigma)$ pairs. The shape of this domain differs according to the indices used and depends on the tolerancing practices of each company [18]. In this paper it is represented on a $\sigma-\delta$ diagram. The shape of the capability domain is triangular in the case where $C_{p}^{(r)}=C_{p k}^{(r)}$ which is the case adopted in this paper. Fig. 1 illustrates the capability domain corresponding to one dimension.

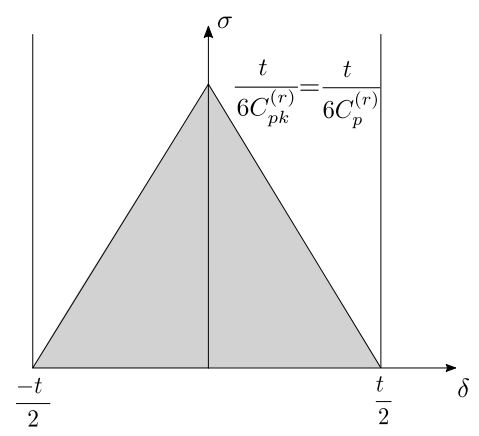

Fig. 1 Representation of the capability domain $V_{D}$ (grey area)

It can presumed that the uncertainty of the process leads to uncertainties in the distribution parameters of the input variables. This will lead to uncertainties in the functional requirement function that will affect in its turn the NCR such as illustrated in Fig. 2 .

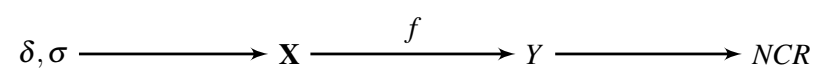

Fig. 2 Uncertainty propagation to the NCR

\section{Proposed procedure for the $\mathrm{KC}$ identification}

The objective of this paper is to define the most contributing dimensions using sensitivity analysis. The procedure to be followed should respect the quality measures and the different requirements given for an industrial problem. In order to perform a sensitivity analysis, it is required to select:

- the input random variables.

- an output quantity, which is expressed in terms of a set of input random variables.

- a suitable sensitivity metrics, as multiple definitions of the sensitivity indices are available.

A model is considered in this study with input and output parameters that need to be selected properly, such as detailed in Section.3.1. The choice of the sensitivity metrics is described in Section.3.2. 


\subsection{Selection of input and output parameters of the model}

Two strategies can be followed to check the importance of the variables. One strategy is to analyse the effect of the dimensions $\boldsymbol{X}$ on the functional characteristics $Y$. An alternative procedure is adopted herein, which consists of investigating the effect of the variable statistical parameters, defined by the vectors $\boldsymbol{\sigma}$ and $\boldsymbol{\delta}$, on the NCR. The statistical parameters are considered because the production of the products is not perfect, therefore there is a variation in the mean shifts and standard deviation of the dimensions. This variability has non negligible impact on the NCR. In addition, their use as input parameters aggregates the effects of the capabilities and of the tolerance intervals. The model has two levels of uncertainties (the parameters $\boldsymbol{\sigma}, \boldsymbol{\delta}$ and the dimensions $\boldsymbol{X}$ ). The functional requirement is expressed in terms of the model uncertainties, it is therefore considered as a variable with two levels of uncertainty. Sensitivity analysis can be applied only to a problem with a single level of uncertainty, therefore the functional requirement cannot be used directly as the output in the sensitivity analysis. However, it is possible to use any statistical quantity derived from the functional requirement such as the mean and standard deviations. It is suggested here to use the NCR that is a function of the statistical parameters. The choice of the NCR, is that in addition to its use by industries as a quality measure, it allows the identification of the interactions for the case of a functional requirement defined by a linear function, unlike the case where the sensitivity analysis is applied on the functional requirement. In addition, the method remains applicable when multiple functional requirements are involved, as the NCR can be computed using system reliability.

\subsection{Choice of the sensitivity analysis method}

The choice of the sensitivity analysis method depends on the required criteria of each problem. First, it is essential to know the existing sensitivity analysis methods that among them, a proper one can be adapted for the identification of the KCs . Saltelli et al. [19] defined the Sensitivity Analysis as the study of how the uncertainty in input parameters can influence the uncertainty in the output of a model. The idea behind the sensitivity analysis is to know the importance of the input design parameters, so the performance of the system can be improved by dealing carefully with these important parameters. Numerous sensitivity analysis methods exist in the literature and are being developed progressively. The challenge of this work is to choose the appropriate approach that is suitable to the problem and that can give consistent results. Sensitivity analysis methods are categorized as local methods and global methods. A detailed review in [20] presents the existing local and global sensitivity methods and then interprets the results of the sensitivity analysis by giving a classical example. Another review in [21] explains the methodological framework of different global sensitivity analysis methods. Local sensitivity considers the influence of the input parameters in the vicinity of a reference point. Mathematically, the local sensitivity analysis consists in estimating the partial derivative with respect to each variable input that characterizes the effect on the random value. In the global sensitivity approach, the impact of an input factor is measured by varying all other input factors [19]. Several approaches corresponding to the global sensitivity analysis exist such as the variance-based and the density-based methods.

- The variance-based methods assume that output variance is a sensible measure of the output uncertainty. They allow the estimation of the main effects of the input variables on the variable output in addition to the total effects which are the interactions between the input variables [22].

- The density-based methods consider the entire distribution without being dependent to a particular moment. They are also known as moment independent methods. Their use is preferable when the variance is not an adequate proxy of uncertainty, this is applicable when the output distribution is multi-modal or highly skewed [23, 24]. This is not the case of our problem.

Among these sensitivity analysis methods, the variance-based method is considered in this article since the concern is to have the sensitivities by considering the variation on the whole domain and not only for a representative point (local sensitivities) and since the variance is considered as a good proxy of the uncertainty. Among the variance-based approaches, Sobol' approach is adopted in this study because of its efficient implementations that are well documented [25,26] and because of the possibility to perform the sensitivity analysis with moderate numerical efforts.

\section{Different steps of the proposed approach}

After the selection of inputs, output and sensitivity method, the procedure to follow can be described as given in Fig.3. The application of the proposed method necessitates the estimation of the NCR which formulation is developed in Sect. 4.1. The sensitivity of the NCR with respect to the variable parameters is performed by applying the Sobol' approach which is one of the most used variance based methos. (Sect.4.2). The use of this method requires the estimation of the NCR $N$ times, where $N$ is the number of the Sobol' iterations which is usually a large number. If the system is linear with dimensions following normal distributions, the NCR has an explicit function, the calculation of the NCR $N$ times is not challenging. However, if the system is non-linear, the 
estimation of the NCR is done using the Monte Carlo technique and there is need to repeat the Monte Carlo simulation $N$ times. Therefore, combining Monte Carlo with the Sobol' sensitivity analysis takes excessive numerical efforts and is time consuming. An alternative way to deal with this problem is the implementation of the re-weighting technique which is similar to the one applied in importance sampling. It is explained in details in Appendix A. Once the sensitivity indices are evaluated, the contributing and non-contribution input parameters can be known. Consequently the KCs can be identified.

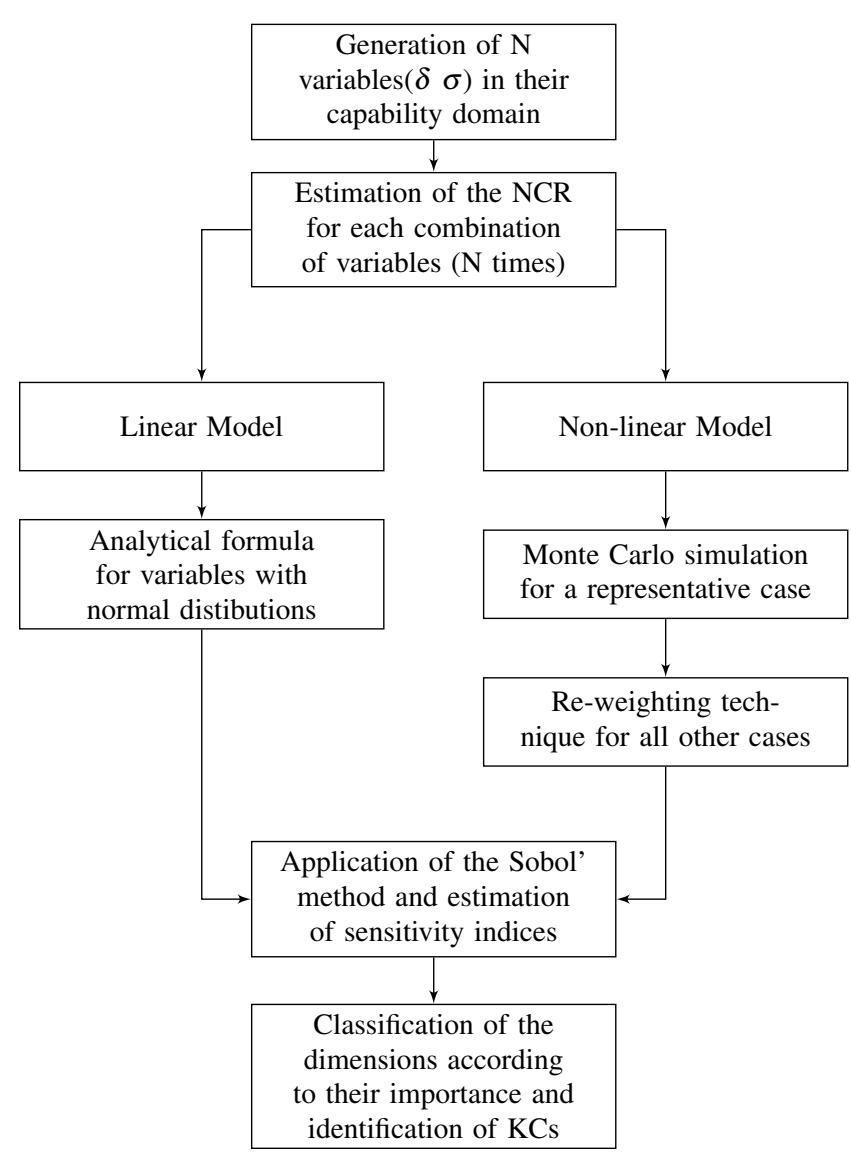

Fig. 3 Steps of the proposed Approach

\subsection{Formulation of the Non-Conformity Rates}

The dimensions are variables following normal distributions that in their turn have variable parameters such as the mean shifts and the standard deviations. These parameters can follow a range of distributions (uniform, normal, etc.). The random vector defining the mean shifts is noted as $\boldsymbol{\delta}=\left(\delta_{1}, \ldots \delta_{n}\right)$ and the one for the standard deviations as $\boldsymbol{\sigma}=\left(\sigma_{1}, \ldots \sigma_{n}\right)$, where $n$ is the number of dimensions. The calculation of the NCR in this work takes into account the variability of the distributions' parameters. The statistical parameters are random variables, therefore the NCR is a random variable as well, it is expressed in terms of $\boldsymbol{\delta}$ and $\boldsymbol{\sigma}$ and can be noted as $\operatorname{NCR}(\boldsymbol{\delta}, \boldsymbol{\sigma})$. The distribution of $\boldsymbol{\sigma}$ and $\boldsymbol{\delta}$ is defined inside the capability domain already discussed in Sect. 2 having a triangular shape. The points that are placed outside this area represent the cases where the capability indices are below their threshold values and such parts should not be used. In industry, the distribution may be identified using the production statistics, but since no other information is available, a uniform distribution is assumed for this study. The proposed approach can also be applied when the Inertial tolerancing [27] or the Process tolerancing [28] is adopted for the estimation of the NCR and the assumption of the capability domains. They also take into account the variability of the statistical parameters.

\section{Estimation of the NCR (general formula)}

When the system is non-linear the NCR is defined similarly to the failure probability which is the integral of the probability density function on the failure domain. A mean shift and a standard deviation is associated to each dimension. The expression of the NCR is given by:

$$
\begin{aligned}
\operatorname{NCR}(\boldsymbol{\delta}, \boldsymbol{\sigma}) & =\int_{g(\boldsymbol{x}) \leq 0} f_{x}(\boldsymbol{x} \mid \boldsymbol{\delta}, \boldsymbol{\sigma}) d x \\
& =\int_{-\infty}^{\infty} I(\boldsymbol{x}) f_{x}(\boldsymbol{x} \mid \boldsymbol{\delta}, \boldsymbol{\sigma}) d x
\end{aligned}
$$

where $f$ is the joint probability density function, and $\mathrm{I}($.$) is$ an indicator function defined by

$I(x)=\left\{\begin{array}{l}1 \text { if } Y \notin\left[L S L_{Y} ; U S L_{Y}\right] \\ 0 \text { otherwise }\end{array}\right.$

where $g(x)$ is the function defining the failure event. The Monte Carlo simulation which is one of the most common sampling technique for the estimation of the failure probability is used for the estimation of the NCR. The estimation of the NCR for a particular case is given by:

$\operatorname{NCR}(\boldsymbol{\delta}, \boldsymbol{\sigma}) \approx \frac{1}{N_{m c}} \sum_{i=1}^{N_{m c}} I\left(\boldsymbol{x}^{(i)}\right)$

where $N_{m c}$ is the sample size and $\boldsymbol{x}^{(i)}$ are samples following a set of independent normal distributions with the mean shifts and the standard deviations. The NCR can be computed using Monte Carlo simulation, even when the problem involves multiple functional requirements. Therefore, the proposed procedure is applicable to problems involving multiple functional requirements.

Estimation of the NCR for a linear case with dimensions following normal distributions 
When the system is linear and the dimensions follow normal distributions, the estimation of the NCR is done using the analytical formula:

$$
\begin{aligned}
N C R(\boldsymbol{\delta}, \boldsymbol{\sigma}) & =\Phi\left(-\frac{\mu_{Y}(\boldsymbol{\delta}, \boldsymbol{\sigma})-L S L_{Y}}{\sigma_{Y}(\boldsymbol{\delta}, \boldsymbol{\sigma})}\right) \\
& +\Phi\left(-\frac{U S L_{Y}-\mu_{Y}(\boldsymbol{\delta}, \boldsymbol{\sigma})}{\sigma_{Y}(\boldsymbol{\delta}, \boldsymbol{\sigma})}\right)
\end{aligned}
$$

where $\Phi$ is the cumulative density function of the standard normal distribution.

4.2 Sensitivity Analysis of the statistical parameters on the NCR

The Sobol' approach is based on the work of Cukier [29]. This approach was first introduced by Sobol' in 1993 [30]. He demonstrated that a square integrable function can be decomposed into a sum of functions. Based on this idea the NCR, which is the studied quantity can be expressed as in Eq.(9).

$$
\begin{aligned}
N C R(\boldsymbol{\delta}, \boldsymbol{\sigma}) & =N C R_{0} \\
& +\sum_{i}^{n} N C R_{i}\left(\delta_{i}, \sigma_{i}\right) \\
& +\sum_{1 \leqslant i \leqslant j \leqslant n} N C R_{i j}\left(\delta_{i} \sigma_{i}, \delta_{j} \sigma_{j}\right) \\
& +\ldots N C R_{12 \ldots n}\left(\delta_{1} \sigma_{1}, \delta_{2} \sigma_{2}, \ldots, \delta_{n} \sigma_{n}\right)
\end{aligned}
$$

where $N C R_{0}=E(N C R)$ is a constant, $N C R_{i}\left(\delta_{i}, \sigma_{i}\right)=$ $\mathrm{E}_{\sim \delta_{i} \sigma_{i}}\left(N C R \mid \delta_{i}, \sigma_{i}\right)$, similar relations are obtained for higher order terms of the decomposition. $E$ is the mathematical expectation. Sobol' indices are based on variance decomposition and determine the part of the variance of the output resulting from each variable. They measure the influence of each input parameter and their interactions to the whole model output variance provided that the parameters of the model are independent. This is called ANOVA decomposition which is obtained by taking into account that the input parameters $\theta_{i}$ are random and independent [31]. The variance of $y$ can be expressed as in Eq.(10).

$V=\sum_{i=1}^{n} V_{i}+\sum \sum_{1 \leqslant i \leqslant j \leqslant n} V_{i j}+\ldots V_{12 \ldots n}$

where $V$ is the total variance of model output which is in this study the $N C R$,

$$
\begin{aligned}
V_{i} & =V\left(N C R_{i}\right)=V\left[E\left(N C R \mid \delta_{i} \sigma_{i}\right)\right] \\
V_{i j} & =V\left[E\left(N C R \mid \delta_{i} \sigma_{i}, \delta_{j} \sigma_{j}\right)\right] \\
& -V\left[E\left(N C R \mid \delta_{i} \sigma_{i}\right)\right]-V\left[E\left(N C R \mid \delta_{j} \sigma_{j}\right)\right]
\end{aligned}
$$

$V_{i}$ is the first order contribution of the $i^{t} h$ model parameter, $V_{i j}$ is the effect of the interaction of the $i^{t h}$ and $j^{t h}$ parameters.
The use of his approach allows the measurement of the firstorder sensitivity index, or the main effect which is the contribution to the output variance of the main effect of one parameter, hence it measures the impact of the variation of an input parameter alone, and averaged over variations in other input parameters. The equation corresponding to the first order index is given by Eq.(11).

$S_{i}=\frac{V_{i}}{V}$

If the value of $S_{i}$ is zero, it can be deduced that the function $N C R$ does not depend on $\delta_{i}, \sigma_{i}$. In another hand if $S_{i}$ is equal to 1 , it means that $N C R$ depends only on $\delta_{i}, \sigma_{i}$. The total Sensitivity index can also be estimated. It measures the interaction of each variable with the other variables [32]. All the terms of the decomposition in Eq.(10) where $i$ is involved are used. It is expressed as:

$S_{T i}=S_{i}+\sum_{j} S_{i j}+\sum_{j, k} S_{i j k}+\ldots$

There is absence of interaction between $X_{i}$ and other variables, if the value of the total effects is equal to the value of the main effects. After the estimation of the NCR $N$ times for both the linear and non-linear cases, the sensitivity analysis based on Sobol' method is performed. The Monte Carlo methods are used for the estimation of the Sobol' indices such as the work of Homma and Saltelli [32]. A more detailed procedure of the calculation of Sobol' indices is described in Appendix B. In this work, the first and the total order indices are estimated. These indices aggregate the contributions of the dimension to the variability of the NCR. Dimensions with high Sobol' indices need additional attention, as they can be associated with large variations of the NCR.

\section{Case Study}

The application of the proposed approach is done on three different cases. The first case is a linear stack-up, the second one is an electrical plug procured by RADIALL having a linearised functional requirement, and the third one is adapted from a Gas Pedal system from Toyota having two non-linear functional requirements. The functional requirements in the three examples are functions of a chain of dimensions. Target values $T_{i}$ are set to each dimension. The dimensions are subject to variations. These variations are expressed by tolerances intervals $t_{i}$ which are assumed to be centred on the corresponding target values. In addition the dimensions follow normal distributions and are subject to capability requirements $C_{p}^{(r)}=C_{p k}^{(r)}$. The Sobol' sensitivity analysis is performed on the NCR with respect to the distribution parameters $\delta$ and $\sigma$ that are set as variables. The 
statistical parameters are expected to vary uniformly in their associated triangular capability domains.

\subsection{Application on a linear stack-up example}

A simple linear stack-up composed of two parts is considered as shown in Fig.4. The functional requirement can be expressed as:

$Y=f(\mathbf{X})=X_{1}+X_{2} \in[9.5 ; 10.5]$

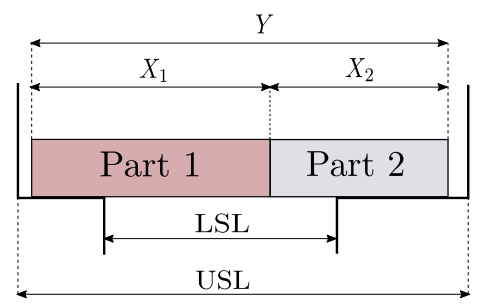

Fig. 4 Simple two-part example

The objective is the identification of variables with a significant influence on the performance of the system. If the local sensitivity is applied that is, the calculation of the partial derivatives, it will be clear that the parts have equal influence on the performance of the system. But this way the tolerance intervals and capability requirements associated to each variable are not taken into consideration. To overcome this problem, the Sobol' sensitivity analysis method is applied. Two procedures can be followed. First the sensitivity analysis is performed on the functional requirement with respect to the dimensions(in this case the distributions' parameters are fixed, $\delta=0$ and $\sigma=\frac{t}{6 C_{p}}$ ). Then the sensitivity analysis is performed on the NCR with respect to the variable parameters characterizing the distribution of the dimensions (mean shifts and standard deviations). A comparison between the two procedures is then done to show the interest of the one adopted in this study. Different cases are considered, they are presented in Table 1. The first case is when the parts have equal input parameters such as the capability requirements and the tolerance intervals. In the second and the third cases the tolerance intervals and capability requirements are changed respectively. The results are shown in Fig. 5. In the first case where the sensitivity analysis is performed on the functional requirement, the two parts have equal impact on the output with no interaction. It is expected since the relation of the functional requirement is linear. In the case, where the sensitivity analysis is performed on the
Table 1 Three different cases of the input data for the linear stack-up example

Case: 1

\begin{tabular}{llll}
\hline Dimensions & $T_{i}$ & $t_{i}$ & $C_{p}^{(r)}=C_{p k}^{(r)}$ \\
\hline$X_{1}$ & 6 & 0.5 & 1 \\
$X_{2}$ & 4 & 0.5 & 1 \\
\hline \multicolumn{4}{l}{ Case: 2} \\
& & \\
\hline Dimensions & $T_{i}$ & $t_{i}$ & $C_{p}^{(r)}=C_{p k}^{(r)}$ \\
\hline$X_{1}$ & 6 & 0.5 & 1 \\
$X_{2}$ & 4 & 0.5 & 0.8 \\
\hline
\end{tabular}

Case: 3

\begin{tabular}{llll}
\hline Dimensions & $T_{i}$ & $t_{i}$ & $C_{p}^{(r)}=C_{p k}^{(r)}$ \\
\hline$X_{1}$ & 6 & 0.8 & 1 \\
$X_{2}$ & 4 & 0.2 & 1 \\
\hline
\end{tabular}

NCR, the interactions between the parts can be measured. The sensitivities of the two parts are no longer independent. When the capability requirements are different, the most important influence is for the part having the less value which is common in the industrial practices. When the tolerance intervals have different values, the most important influence is for the part having the highest value. This example shows the interests of the method:

- it detects the interactions between input parameters regardless the linearity of the model.

- it aggregates the effects of the tolerance intervals and of the capability requirements.

\subsection{Application on the Electrical plug from RADIALL}

The application of the method is done also on a linearised function. The case from RADIALL is an electrical plug. Fourteen dimensions are involved in the dimensional chain following the relation given in Eq.(14).

$Y=f(\boldsymbol{X}) \approx a_{0}+\sum_{0}^{14} a_{i} X_{i}$

The functional requirement is that the misalignment of the tip of the electric plug should not exceed a threshold value such as shown in Fig. 6 (The example is provided by an industrial partner, we did not have access to the CAD model, the figure is included as an illustration only). This functional requirement is set as critical according to an analysis based on FMECA. All the dimensions involved are considered as 


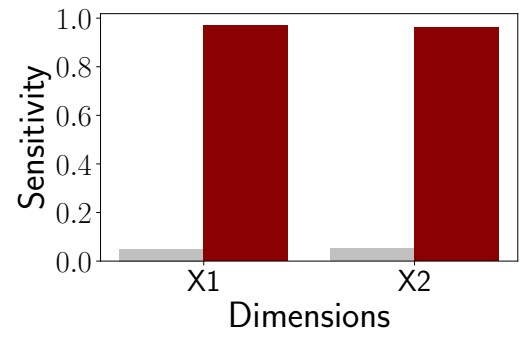

(a) Case 1

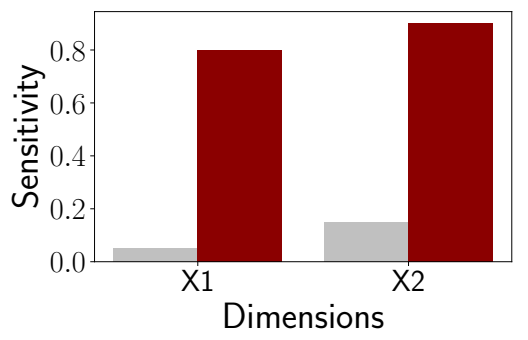

(b) Case 2

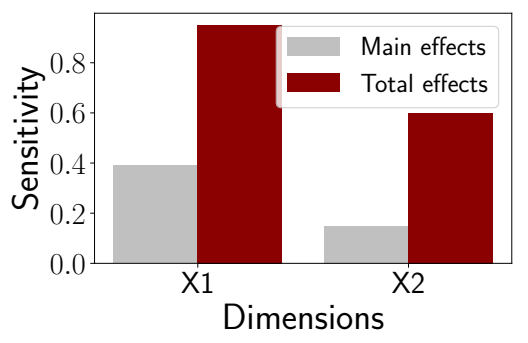

(c) Case 3

Fig. 5 First and Total order indices based on Sobol' sensitivity analysis on the NCR

KCs when the design procedure used at RADIALL is applied. The input data of the problem is given in Table 2. The purpose of this example to be able to classify the dimensions regarding their importance on the performance of the system. The first and total sensitivity indices are estimated by the application of the Sobol' sensitivity analysis on the NCR with respect to the statistical parameters.

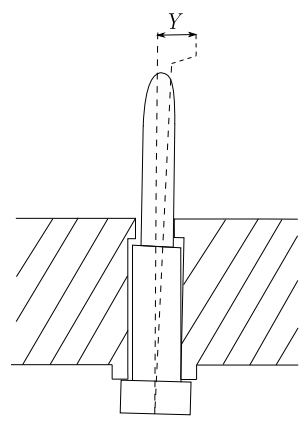

Fig. 6 Simplified Electrical Plug
Table 2 Coefficients, target values, tolerance intervals and capability requirements for RADIALL case input parameters

\begin{tabular}{lllll}
\hline Dimensions & $a_{i}$ & $T_{i}$ & $t_{i}$ & $C_{p}^{(r)}=C_{p k}^{(r)}$ \\
\hline X1 & -0.04 & 10.53 & 0.2 & 1.1 \\
X2 & -0.5 & 0.1 & 0.2 & 1.1 \\
X3 & -0.5 & 0 & 0.06 & 1.1 \\
X4 & 1.14 & 0.643 & 0.015 & 1.1 \\
X5 & 0.91 & 0 & 0.06 & 1.1 \\
X6 & 0.91 & 0.72 & 0.04 & 1.1 \\
X7 & $1.10^{-4}$ & 1.325 & 0.05 & 1.1 \\
X8 & 0.05 & 0.75 & 0.04 & 1.1 \\
X9 & $1.10^{-4}$ & 0 & 0.04 & 1.1 \\
X10 & 0.13 & 3.02 & 0.06 & 0.86 \\
X11 & -1.4 & 0.72 & 0.04 & 0.86 \\
X12 & -1.15 & 0 & 0.04 & 0.86 \\
X13 & -0.9 & 0.97 & 0.04 & 0.86 \\
X14 & 0.13 & 0.4 & 0.06 & 0.86 \\
\hline
\end{tabular}

The results are shown in Fig. 7. It can be deduced that the variation of the statistical parameters of $X_{2}$ and $X_{5}$ has the highest contribution on the value of the NCR. Looking at the main effects, $50 \%$ of the variation of the NCR is due to the variation in the parameters corresponding to $X_{5}$ and almost $12 \%$ due to $X_{2}$. The total effects show that there are interactions between the input parameters and most probably between $X_{2}, X_{5}, X_{11}, X_{12}$ and $X_{13}$. Based on these results, the mentioned dimensions can be set as KCs.

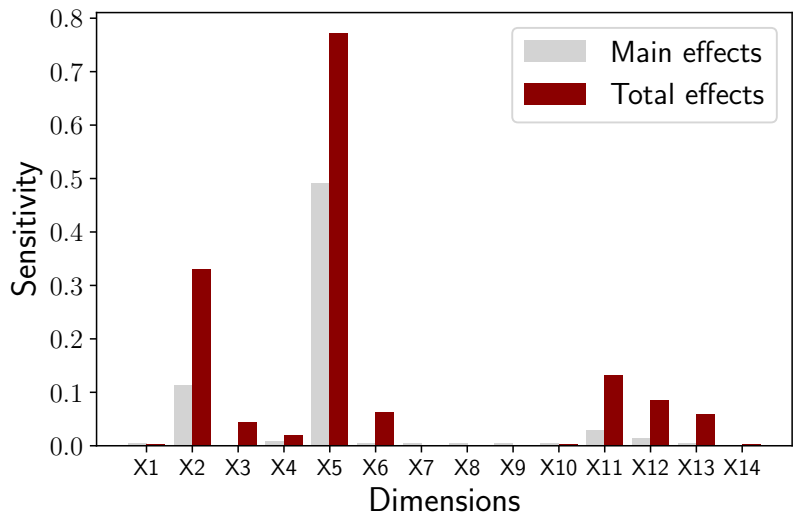

Fig. 7 First and Total order Sobol' Sensitivity indices for RADIALL case dimensions 


\section{Validation of the results}

To validate the results of this approach, the dimensions that are set as KCs are grouped together. The problem is to check the sensitivity of the NCR to the KCs alone and to the NonKCs. Figure 8 shows the percentage of the variability of the NCR caused by the variability of the KCs, of the non-KCs and their interactions. The results show that when there is a variation in the NCR, $88 \%$ of this variation is caused by the dimensions set as $\mathrm{KCs}$, only $4 \%$ from the non-KCs, and the rest from their interaction. Relying on these values, it can be deduced that the choice of the KCs is relevant since they have an important contribution on the NCR (more than $80 \%)$.

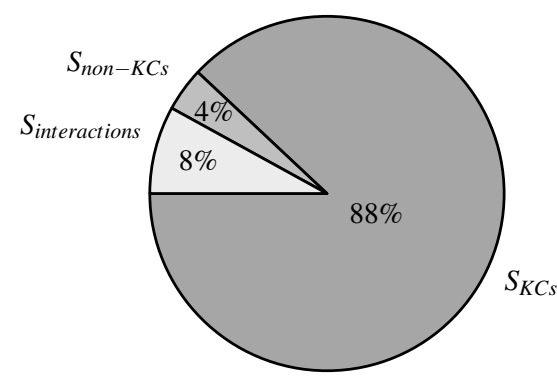

Fig. 8 Sensitivity of the NCR to the KCs, to the non-KCs and to their interactions

The use of this approach allows the aggregation of the coefficients $a_{i}$ and the tolerance intervals and the capability requirements and allows to see the interactions between the dimensions and to take into consideration the mean shifts and the variation of the standard deviations.

\subsection{Application on the Toyota Pedal case}

Another application of the proposed method is on a Toyota gas Pedal system shown in Fig. 9 and inspired from [33]. Two functional requirements should be satisfied in this example. The moment and the bending stress, give respectively in Eq. 15 and Eq. 16, have maximum values that should not be exceeded $\left(M_{\max }=500 \mathrm{Nmm}\right.$ and $\sigma_{\max }=50 \mathrm{MPa}$ following a normal distribution with $\pm 5 M P a$ its estimated variation). The interest of this example is to show that the choice of this method overcomes the problem faced to identify KCs when the more than one functional requirement exist in the system. The input data are given in Table 3. The sensitivity of the NCR with respect to the dimensional variables is studied. A product is considered non-conform when the corresponding moment or the bending stress does not lie in the required range of values.
The Monte Carlo method is used to calculate the NCR for a reference case where the mean of each variable is equal to its target value, that the zero mean shifts, and where the standard deviations are maximum. The re-weighting technique is then used to calculate the NCR associated with other cases where the mean shifts and standard deviations are variables.

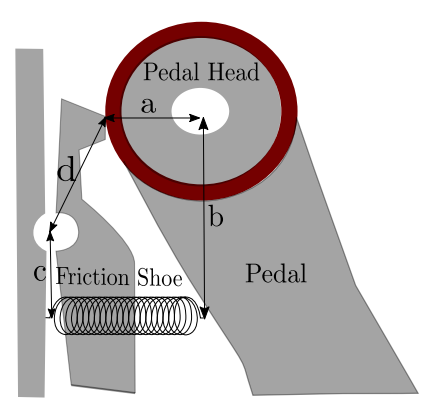

Fig. 9 Toyota Gas Pedal System

$$
\begin{gathered}
M=F_{\text {spring }} \cdot\left(b-\frac{c}{d} \cdot \mu_{f} \cdot a\right) \\
\sigma_{b}=\frac{6 \cdot F_{\text {spring }} \cdot c}{w \cdot h^{2}} \\
\text { Where } F_{\text {spring }}=k \cdot s
\end{gathered}
$$

Table 3 Target values, tolerance intervals and capability requirements for Toyota gas pedal input parameters.

\begin{tabular}{llll}
\hline Dimensions & $T_{i}$ & $t_{i}$ & $C_{p}^{(r)}=C_{p k}^{(r)}$ \\
\hline$a$ & 10 & 0.1 & 1.3 \\
$b$ & 16 & 0.1 & 1.3 \\
$c$ & 11 & 0.12 & 1.3 \\
$d$ & 8 & 0.1 & 1.3 \\
$s$ & 16 & 0.2 & 1 \\
$w$ & 4 & 0.08 & 1 \\
$h$ & 5 & 0.08 & 1 \\
\hline
\end{tabular}

The spring stiffness $k$ and the coefficient of friction $\mu_{f}$ are also random variables following uniform distributions. $k$ varies in the interval [3.5;4.5] and $\mu_{f}$ varies in the interval [0.65; $0.75]$. They are considered for the estimation of the NCR, however, they are excluded from the sensitivity analysis since they are not dimensions and the designers cannot impose any changes to these variables. The results of the analysis are shown in Fig. 10. It is noticeable that the main effects of the dimensions are negligible unlike the total effects that have significant values for all the dimensions. It can be concluded that the variation of the NCR is caused by the interactions between the input variables. All the dimensions are set 
as $\mathrm{KCs}$ since they are all contributing and it is not possible to prioritize one on another .

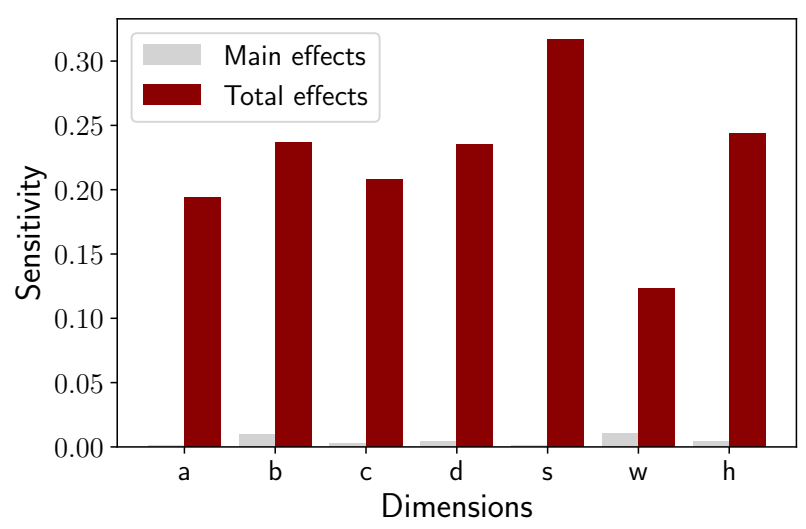

Fig. 10 First and total order Sobol' indices for Toyota Gas Pedal dimensions

\section{Conclusion}

This paper presents a design approach for the identification of $\mathrm{KCs}$ in the critical dimensional chains. It allows the reduction of the number of KCs with respect to the existent practices in the early design stage. Therefore, the efforts dedicated to check the $\mathrm{KCs}$ are reduced and consequently the manufacturing production costs. The application of this approach allows the production with a reduced cost but without losing the quality of the product. This proposed method is a numerical method that takes into account the tolerances during the design stage and the variation of the manufacturing processes. Therefore it establishes a link between the work in the design offices from one side and the production processes from another side and enables them to work in an interactive environment. As a consequence it allows the interactivity between the designers and the manufacturing engineers. In addition, it prevents derogations after the production (accepting the non-compliant products to be delivered). The sensitivity analysis is performed on the NCR to identify the dimensions with high importance. The Sobol' global sensitivity method is adopted in this context. For a better accuracy, the NCR is estimated by taking into consideration the variability of the input parameters associated to the dimensions such as the mean shifts and the standard deviations characterising their distributions. This is a sensitivity analysis performed on two levels of uncertainty. The application of this approach is done first on a linear stack-up case with only two parts. The results show that the proposed approach can show the interactions between parts even for a linear functional characteristic knowing that the NCR is the quantity studied. Another reason for the use of this ap- proach is that it aggregates the input parameters, such as the tolerance intervals, the capability requirements and the multiplying coefficients. This is illustrated in the example of the electrical plug with a linearised functional characteristic. Furthermore, one other advantage of this approach is that it can be applied for the case where more than one functional requirement is needed. The application example of the Toyota gas pedal illustrates this idea. It is also important to mention that it is a model free method, that is can be performed for the linear and the non-linear systems. The application of the proposed approach on an over-constrained system is a perspective of this work. In addition, the parts are considered as rigid bodies so only dimension variations are taken into account. Form and location variations can be considered in later works. 


\section{A}

\section{Re-weighting technique for the estimation of the NCR}

The re-weighting technique consists of considering one representative case where the mean values are assumed equal to the target value and the standard deviations are assumed to have their maximum values. A Monte Carlo simulation is conducted for the estimation of the NCR for this particular case such as given in Eq. 1.

$N C R\left(\boldsymbol{\delta}_{\mathbf{0}}, \boldsymbol{\sigma}_{\mathbf{0}}\right)=\int_{-\infty}^{\infty} I(\boldsymbol{x}) f_{x}\left(\boldsymbol{x} \mid \boldsymbol{\delta}_{\mathbf{0}}, \boldsymbol{\sigma}_{\mathbf{0}}\right) d x$

The re-weighting technique is applied to estimate the NCR for the other cases of different means and standard deviations. Equation (2) is the expression of the NCR for another case of different mean shifts and standard deviations. It is then multiplied by the ratio of the density function defined for the reference case to the same density function so that it is equal to 1, this is given by Eq. (3).

$N C R\left(\boldsymbol{\delta}_{1}, \boldsymbol{\sigma}_{1}\right)=\int_{-\infty}^{\infty} I(\boldsymbol{x}) f_{x}\left(\boldsymbol{x} \mid \boldsymbol{\delta}_{\mathbf{1}}, \boldsymbol{\sigma}_{\mathbf{1}}\right) d x$

$$
\begin{aligned}
N C R\left(\boldsymbol{\delta}_{1}, \boldsymbol{\sigma}_{1}\right)= & \int_{-\infty}^{\infty} I(\boldsymbol{x}) f_{x}\left(\boldsymbol{x} \mid \boldsymbol{\delta}_{1}, \boldsymbol{\sigma}_{1}\right) \frac{f_{x}\left(\boldsymbol{x} \mid \boldsymbol{\delta}_{\mathbf{0}}, \boldsymbol{\sigma}_{0}\right)}{f_{x}\left(\boldsymbol{x} \mid \boldsymbol{\delta}_{\mathbf{0}}, \boldsymbol{\sigma}_{\mathbf{0}}\right)} d x \\
& =\int_{-\infty}^{\infty} I(\boldsymbol{x}) f_{x}\left(\boldsymbol{x} \mid \boldsymbol{\delta}_{\mathbf{0}}, \boldsymbol{\sigma}_{\mathbf{0}}\right) \frac{f_{x}\left(\boldsymbol{x} \mid \boldsymbol{\delta}_{\mathbf{1}}, \boldsymbol{\sigma}_{1}\right)}{f_{x}\left(\boldsymbol{x} \mid \boldsymbol{\delta}_{\mathbf{0}}, \boldsymbol{\sigma}_{\mathbf{0}}\right)} d x
\end{aligned}
$$

The estimation of the NCR can be given by:

$N C R\left(\boldsymbol{\delta}_{1}, \boldsymbol{\sigma}_{1}\right) \approx \frac{1}{N_{m c}} \sum_{i=1}^{N_{m c}} I\left(\boldsymbol{x}^{(i)}\right) \frac{f_{x}\left(\boldsymbol{x}^{(i)} \mid \boldsymbol{\delta}_{1}, \boldsymbol{\sigma}_{1}\right)}{f_{x}\left(\boldsymbol{x}^{(i)} \mid \boldsymbol{\delta}_{\mathbf{0}}, \boldsymbol{\sigma}_{\mathbf{0}}\right)}$

The new NCR can be deduced from the reference one that is already estimated from Eq.(1). After the application of this approach, there is no need to generate new samples for each case where the density function parameters vary. The same sample points are kept from the failure region that provide non-zero values of the indicator function $I(\boldsymbol{x})$ and then multiplied by the weights.

\section{B}

\section{Computation of Sobol' sensitivity indices}

The common way to compute the Sobol' indices is to use what is known as the brute force Monte Carlo approach [19,?]. Two matrices $\mathrm{A}$ and $\mathrm{B}$ are generated comprising the input parameters denoted respectively $a_{j i}$ and $b_{j i}$ where $i=1, \ldots, n$ and $j=1 \ldots N . n$ is the number of input variables and $N$ is the number of generated samples.

$A=\left[\begin{array}{ccccc}a_{11} & \cdots & a_{1 i} & \cdots & a_{1 n} \\ \vdots & \cdots & \vdots & \cdots & \vdots \\ a_{j 1} & \cdots & a_{j i} & \cdots & a_{j n} \\ \vdots & \cdots & \vdots & \cdots & \vdots \\ a_{N 1} & \cdots & a_{N i} & \cdots & a_{N n}\end{array}\right] B=\left[\begin{array}{ccccc}b_{11} & \cdots & b_{1 i} & \cdots & b_{1 n} \\ \vdots & \cdots & \vdots & \cdots & \vdots \\ b_{j 1} & \cdots & b_{j i} & \cdots & b_{j n} \\ \vdots & \cdots & \vdots & \cdots & \vdots \\ b_{N 1} & \cdots & b_{N i} & \cdots & b_{N n}\end{array}\right]$

A matrix $A_{B}$ is defined, having all the columns of matrix $A$ except the ith column from matrix $B$. The same concept is applied on a matrix called $B_{A}$ such that all its columns are from matrix $B$ except the ith column that comes from matrix $A$. They are presented as:

$A_{B}^{(i)}=\left[\begin{array}{ccccc}a_{11} & \cdots & b_{1 i} & \cdots & a_{1 n} \\ \vdots & \cdots & \vdots & \cdots & \vdots \\ a_{j 1} & \cdots & b_{j i} & \cdots & a_{j n} \\ \vdots & \cdots & \vdots & \cdots & \vdots \\ a_{N 1} & \cdots & b_{N i} & \cdots & a_{N n}\end{array}\right] B_{A}^{(i)}=\left[\begin{array}{ccccc}b_{11} & \cdots & a_{1 i} & \cdots & b_{1 n} \\ \vdots & \cdots & \vdots & \cdots & \vdots \\ b_{j 1} & \cdots & a_{j i} & \cdots & b_{j n} \\ \vdots & \cdots & \vdots & \cdots & \vdots \\ b_{N 1} & \cdots & a_{N i} & \cdots & b_{N n}\end{array}\right]$

The first order index is calculated from the matrices $A$ with $B_{A}^{(i)}$ by applying the following formula:

$\operatorname{Var}_{X_{i}}\left[E\left[Y \mid X_{i}\right]\right]=\frac{1}{N} \sum_{j=1}^{N} f(A)_{j} f\left(B_{A}^{(i)}\right)-f_{0}^{2}$

where

$f_{0}=\frac{1}{N} \sum_{j=1}^{N} f(A)_{j}$

The first order sensitivity index is computed as shown in Eq.B.3

$S_{i}=\frac{V_{i}}{V}$

The total order index is calculated from the matrices $A$ and $A_{B}^{(i)}$ by applying the following formula:

$\operatorname{Var}_{X_{i}}\left[E\left[Y \mid X_{\sim i}\right]\right]=\frac{1}{N} \sum_{j=1}^{N} f(A)_{j} f\left(A_{B}^{(i)}\right)-f_{0}^{2}$

$S_{T i}=1-\frac{V_{\sim i}}{V}$ 


\section{References}

1. S Khodaygan. An interactive method for computer-aided optimal process tolerance design based on automated decision making. International Journal on Interactive Design and Manufacturing, 13(1):349-364, 2019.

2. Xavier Fischer, Alain Daidie, Benoit Eynard, and Manuel Paredes, editors. Research in Interactive Design: Mechanics, Design Engineering and Advanced Manufacturing, volume 4. Springer, 2012.

3. Ilham H. Ibrahim and Constantin Chassapis. An interactive variation risk management environment to assess the risk of manufacturing variations. International Journal on Interactive Design and Manufacturing, 11(3):597-608, 2017.

4. Anna C. Thornton. Variation Risk Management. John Wiley \& Sons, 2004.

5. The Boeing Group. Advance Quality Systems Tools - AQS D19000-1. 1998.

6. Reliability Analysis Center. Failure mode, effects, and criticality analysis. Microelectronics Reliability, 7(3):268, 1968.

7. A Van Hoecke. Hierarchization of characteristics applied to the component approval strategy. Procedia CIRP, 10:238-250, 2013.

8. Anna C Thornton. Mathematical framework for the key characteristic process. Research in Engineering Design - Theory, Applications, and Concurrent Engineering, 11(3):145-157, 1999.

9. J Y Dantan, A Hassan, A Etienne, A Siadat, and P Martin. Information modeling for variation management during the product and manufacturing process design. International Journal on Interactive Design and Manufacturing (IJIDeM), 2(2):107-118, 2008.

10. Xin Han, Rong Li, Jian Wang, Shengfeng Qin, and Guofu Ding. Identification of key design characteristics for complex product adaptive design. International Journal of Advanced Manufacturing Technology, 95(1-4):1215-1231, 2018.

11. Julia Stuppy and Halard Meerkamm. Tolerance analysis of a crank mechanism by taking into account different kinds of deviation. In Giordano, M., Villeneuve, F., Mathieu, L., editor, Proceedings of the 11th CIRP International Conference on Computer Aided Tolerancing, Annecy, 2009.

12. Yann Ledoux and Denis Teissandier. Tolerance analysis of a product coupling geometric and architectural specifications in a probabilistic approach. Research in Engineering Design, 24(3):297311,2013

13. Tobias Eifler, Johannes Mathias, Engelhardt Roland, Kloberdanz Hermann, Birkhofer Herbert, and Andrea Bohn. Evaluation of solution variants in conceptual design by means of adequate sensitivity indices. Dispositions in Teacher Education: A Global Perspective, (August):199-209, 2011.

14. Philipp Ziegler and Sandro Wartzack. A statistical method to identify main contributing tolerances in assemblability studies based on convex hull techniques. Journal of Zhejiang UniversitySCIENCE A, 16(5):361-370, 2015.

15. P. Beaucaire, N. Gayton, E. Duc, and J. Y. Dantan. Statistical tolerance analysis of a mechanism with gaps based on system reliability methods. Procedia CIRP, 10(12):2-8, 2013.

16. Jean-yves Dantan, Nicolas Gayton, Alain Etienne, Ahmed Jawad Qureshi, Jean-yves Dantan, Nicolas Gayton, Alain Etienne, Ahmed Jawad, and Qureshi Mathematical. Mathematical issues in mechanical tolerance analysis To cite this version : HAL Id : hal-00756539. 2012.

17. Dc Montgomery. Introduction to statistical quality control. John Wiley \& Sons, 2009.

18. Vijay Srinivasan. ISO Deliberates Statistical Tolerancing, pages 77-87. Springer US, Boston, MA, 1998.

19. Andrea Saltelli, Marco Ratto, Stefano Tarantola, and Francesca Campolongo. Sensitivity analysis practices: Strategies for modelbased inference. Reliability Engineering \& System Safety, 91(1011):1109-1125, 2006.
20. Emanuele Borgonovo and Elmar Plischke. Sensitivity analysis A review of recent advances. European Journal of Operational Research, 248(3):869-887, 2016.

21. Bertrand Iooss, Paul Lemaître, Bertrand Iooss, and Paul Lemaître. A review on global sensitivity analysis methods. C. Meloni and G. Dellino. In Uncertainty management in Simulation- Optimization of Complex Systems: Algorithms and Applications. Springer 2015, 2015.

22. Andrea Saltelli, Paola Annoni, Ivano Azzini, Francesca Campolongo, Marco Ratto, and Stefano Tarantola. Variance based sensitivity analysis of model output. Design and estimator for the total sensitivity index. Computer Physics Communications, 181(2):259-270, 2010.

23. Emanuele Borgonovo, William Castaings, and Stefano Tarantola. Moment Independent Importance Measures: New Results and Analytical Test Cases. Risk Analysis, 31(3):404-428, 2011.

24. Francesca Pianosi and Thorsten Wagener. A simple and efficient method for global sensitivity analysis based on cumulative distribution functions. Environmental Modelling \& Software, 67:1-11, 2015.

25. I M Sobol. Global sensitivity indices for nonlinear mathematical models and their Monte Carlo estimates. Mathematics and Computers in Simulation, 55(1):271-280, 2001.

26. S. Kucherenko and S. Song. Different numerical estimators for main effect global sensitivity indices. Reliability Engineering and System Safety, 165(February):222-238, 2017.

27. Maurice Pillet. Inertial tolerancing. TQM Magazine, 16(3):202209, 2004.

28. E M Mansoor. The application of probability to tolerances Used in Engineering Designs. Proceeding of the institution of Mechanical Engineers, 178.1(1):p28--51, 1963.

29. R I Cukier, C M Fortuin, K E Shuler, A G Petschek, and J H Schaibly. Study of the sensitivity of coupled reaction systems to uncertainties in rate coefficients. I Theory. Journal of Chemical Physics, 59(8):3873-3878, 1973.

30. I M Sobol. Sensitivity analysis for nonlinear mathematical models. Math. Model. Computer.Exp, 1(4):407-414, 1993.

31. B Efron and C Stein. The Jackknife Estimate of Variance. The Annals of Statistics, 9(3):586-596, 1981.

32. T Homma and Andrea Saltelli. Importance measures in global sensitivity analysis of nonlinear models. Reliability Engineering \& System Safety, 52:1-17, 1996.

33. Simon Moritz Göhler, Tobias Eifler, and Thomas J Howard. Robustness Metrics: Consolidating the Multiple Approaches to Quantify Robustness. Journal of Mechanical Design, 138(11):111407, 2016. 